This is the post print version of the article, which has been published in Reactive and functional polymers. 2018, 124, 29-39. http://dx.doi.org/10.1016/j.reactfunctpolym.2017.12.019.

\title{
Soft hydrazone crosslinked hyaluronan- and alginate-based hydrogels as 3D supportive matrices for human pluripotent stem cell-derived neuronal cells
}

\author{
Jennika Karvinen ${ }^{\text {la }}$, Tiina Joki ${ }^{\mathrm{b}}$, Laura Ylä-Outinen ${ }^{\mathrm{b}}$, Janne T. Koivisto ${ }^{\mathrm{a}, \mathrm{b}}$, Susanna \\ Narkilahti ${ }^{\mathrm{b}}$, Minna Kellomäki ${ }^{\mathrm{a}, \mathrm{b}}$ \\ ${ }^{a}$ BioMediTech Institute and Faculty of Biomedical Sciences and Engineering, Tampere University of Technology, \\ Korkeakoulunkatu 3, FI-33101 Tampere, Finland \\ ${ }^{b}$ BioMediTech Institute and Faculty of Medicine and Life Sciences, University of Tampere, Arvo Ylpön katu 34, \\ FI-33520 Tampere, Finland
}

\begin{abstract}
Regenerative medicine, especially cell therapy combined with a supportive biomaterial scaffold, is considered to be a potential treatment for various deficits in humans. Here, we have produced and investigated the detailed properties of injectable hydrazone crosslinked hyaluronanpolyvinyl alcohol (HA-PVA) and alginate-polyvinyl alcohol (AL-PVA) hydrogels to be used as a supportive biomaterial for 3D neural cell cultures. To the best of our knowledge, this is the first time the polymerization and properties of hydrazone crosslinked AL-PVA hydrogel have been reported. The effect of the degree of substitution and molecular weight of the polymer components as well as the polymer concentration of the hydrogel on the swelling, degradation and mechanical properties of the hydrogels is reported. Furthermore, we studied the effect of the above parameters on the growth of human pluripotent stem cell-derived neuronal cells. The most neural cell supportive HA-PVA hydrogel was composed of high molecular weight HA component with brain-mimicking mechanical properties and decreased polymer concentration. AL-PVA hydrogel, with stiffness quite similar to brain tissue, was also shown to be similarly supportive. Neuronal spreading and 3D network formation was enhanced inside the softest hydrogels.
\end{abstract}

Keywords: hyaluronan, alginate, hydrazone, hydrogel, 3D neuronal culture

\section{Introduction}

Traumas and deficits in the human central nervous system (CNS) may have a permanent effect on the functionality of the patient and the prognosis in many cases is poor. Moreover, human CNS, as an organ, suffers from low inbuilt regenerative capacity, which makes healing with traditional medicine (drugs and surgical operations) insufficient. As a result, regenerative medicine is considered to be a potential treatment for CNS deficits. Regenerative medicine aims to restore normal functionality by enhancing the regeneration of tissue or by replacing the damaged parts with engineered biological transplants. One such strategy is cell therapy combined with a supportive biomaterial scaffold.

\footnotetext{
${ }^{1}$ Corresponding author: E-mail:jennika.karvinen@tut.fi, Tel.: +358 408490988
} 
Biomaterial scaffold should fulfill specific criteria when they are used for neural tissue engineering with the aim of neuronal network regeneration. For example, the scaffolds should have similar mechanical properties to those of the brain or spinal cord, they should allow the infiltration of cells and axons, they should allow the transportation of nutrients and metabolites, they should integrate with the host tissue, they should exhibit a suitable degradation rate without any harmful degradation products, and they should not induce inflammatory and glial scar formation [1]. Based on these requirements, polymer-based hydrogels can be considered to be suitable biomaterial candidates for neural tissue engineering. A soft, hydrated form of three-dimensional crosslinked hydrogels resembles that of naturally occurring living tissue. The porous nature of hydrogels enables the transportation of waste, oxygen and nutrients. The mechanical and physical properties of hydrogels are tunable making it easier to mimic the living tissue [2]. Most hydrogels are also considered to be cyto- and biocompatible materials.

When designing hydrogels for this kind of application, a thorough knowledge of their various properties is important. Hydrogels have variable mechanical, physical and degradation properties that can be controlled, for example, by altering the molecular weight $\left(\mathbf{M}_{w}\right)$, the chemical structure and the number of available crosslinkable groups in the polymer, the ratio of gel components, the amount of water, and the crosslinking method. It is well known that, for example, crosslinking density can affect mechanical, swelling and degradation properties and the functionality of hydrogels. The difficulty lies in the altering of properties individually when needed without affecting the others [3]. Further modification of hydrogels can be carried out by incorporating extracellular matrix (ECM) molecules (collagen, laminin, etc.) or peptides to provide more anchoring sites for the cells [4, 5].

Numerous natural and synthetic polymer-based hydrogels have been used as scaffolds for the 3D culture of neural lineage cells as reviewed by, for example, Murphy, et al. 2017 [2]. Two widely used polysaccharide polymers used in neural research are hyaluronan (HA) [6, 7] and alginate [8, 9]. HA is an anionic and hydrophilic polysaccharide composed of $\beta-1,4-\mathrm{D}$-glucuronic acid and $\beta-1,3-\mathrm{N}$-acetyl-D-glycosamine residues that is a major glycosaminoglycan component in the ECM of the brain. HA plays a vital role in the development of the CNS, and it is particularly abundant in the fetal brain and the surrounding immature neurons during differentiation in the spinal cord [10]. HA is produced by almost all the members of the animal kingdom as well as certain members of the streptococci species. It has a relatively simple repetitive chemical structure. The carboxyl and hydroxyl groups allow specific modification and the introduction of functional groups for crosslinking. Alginate (AL) is an anionic and hydrophilic polysaccharide composed of 1,4-linked $\beta$-D-mannuronic acid (M) and $\alpha$-L-guluronic acid (G) residues that is obtained from brown algae. It is structurally similar to ECM. AL is inherently non-biodegradable, but it can be made degradable, for example, by replacing the divalent cations with monovalent cations or by oxidation [11]. The properties of natural polymer-based hydrogels can be improved and widened by combining them with a synthetic polymer such as polyvinyl alcohol (PVA). PVA is a hydrophilic polymer with good chemical, thermal and mechanical stability. Hydroxyl groups in the structure provide chemical versatility that enables further modification and functionalization [12]. Biocompatible PVA hydrogels have been used quite widely in several biomedical applications [13]. PVA has also been approved by the Food and Drug Administration (FDA) and Conformité Européenne (CE) for clinical use in humans [14].

HA-PVA and AL-PVA hydrogels can be fabricated by using hydrazone crosslinking, which is an aldehyde-hydrazide coupling reaction. Hydrazone crosslinking belongs to the group of pseudo click chemistry reactions together with the Michael addition reaction (pseudo is usually characterized by moderate orthogonality). The reactions are versatile, simple and reversible, 
and they have high reactivity and yield. Furthermore, no toxic reagents or side-products are produced [15]. This crosslinking method also enables the fabrication of injectable hydrogels. The injectability of the hydrogels is desirable for when the hydrogels are eventually transplanted into the injury site. To date, there have been several studies carried out on hydrazone crosslinked HA-PVA hydrogels [16, 17]. The focus of these studies has, however, been on other areas of soft tissue engineering. To the best of our knowledge, this is the first time the polymerization and properties of the hydrazone crosslinked AL-PVA hydrogel have been reported.

In this study, we have produced injectable hydrazone crosslinked HA-PVA and AL-PVA hydrogels with variable properties. We studied the effect of the degree of substitution (DS\%), the molecular weight of the polymer components and the polymer concentration of the hydrogel on swelling, degradation and the mechanical properties of the hydrogels. We also studied the effects of the above parameters on the growth of human pluripotent stem cell-derived neuronal cells.

\section{Materials and Methods}

\subsection{Materials and general methods}

Hyaluronic acid sodium salt $\left(\mathrm{M}_{w}=1.5 \times 10^{5} \mathrm{~g} / \mathrm{mol}\right)$ was purchased from Lifecore (Chaska, $\mathrm{MN}$, USA). Hyaluronic acid sodium salt from streptococcus equi $\left(\mathrm{M}_{w}=1.5-1.8 \times 10^{6} \mathrm{~g} / \mathrm{mol}\right)$, polyvinyl alcohol $\left(\mathrm{M}_{w}=27000 \mathrm{~g} / \mathrm{mol}, 98.0-98.8 \%\right.$ hydrolyzed), t-butyl carbazate (TBC), 1,1'carbonyldiimidazole (CDI), glycine ethyl ester hydrochloride, hydrazine solution (35 wt.\% in $\mathrm{H}_{2} \mathrm{O}$ ), 1-hydroxybenzotriazole (HOBt), alginic acid sodium salt from brown algae (low viscosity), picrylsulfonic acid solution (5-\% (w/v) in $\mathrm{H}_{2} \mathrm{O}$, TNBS), hyaluronidase from bovine testes (Type I-S, 400-1000 units/mg solid), ethylene glycol, dimethyl sulfoxide (DMSO), deuterium oxide (99.9 atom \% D, contains 0.05 wt.\% 3-(trimethylsilyl)-propionic-2,2,3,3-d4 acid, sodium salt), sodium cyanoborohydride $\left(\mathrm{NaBH}_{3} \mathrm{CN}\right)$, sucrose, sodium periodate, sodium acetate, acetic acid, sodium tetraborate decahydrate and boric acid were purchased from Sigma-Aldrich (St. Louis, MO, USA). Triethylamine was purchased from J.T. Baker (The Netherlands). Acetate buffer was prepared from sodium acetate and acetic acid, and borate buffer was prepared from sodium tetraborate decahydrate and boric acid. All solvents used were of analytical quality. Milli-Q water was used in synthesis and determinations. Dialysis membranes (Spectra/Por ${ }^{\circledR}$ cut-off 1000 and 25,000 g/mol) were purchased from Spectrum Laboratories, Inc. (Rancho Dominguez, CA, USA).

Cell culture reagents DMEM/F12, Neurobasal, GlutaMax, B27, N2 and penicillin/streptomycin were purchased from Thermo Fisher Scientific (Waltham, MA, USA), and basic fibroblast growth factor (FGF2) from Bio-Techne (Minneapolis, MN, USA). Rabbit anti-microtubule associated protein 2 (MAP-2, AB5622) was purchased from Merck Millipore (Darmstadt, Germany), monoclonal mouse anti-B-tubulin III (T8660) was purchased from Sigma-Aldrich (St. Louis, MO, USA) and Alexa Fluor 488 or 568 conjugated secondary antibodies (1:400) from Thermo Fisher Scientific. VECTASHIELD mounting media containing 4',6-diamidino-2-phenylindole (DAPI) was purchased from Vector Laboratories (Peterborough, United Kingdom).

Nuclear Magnetic Resonance (NMR) spectroscopy experiments were measured with a Varian Mercury $300 \mathrm{MHz}$ NMR Spectrometer (Palo Alto, USA). Polymer samples (5 mg) were dissolved deuterium oxide $(600 \mu \mathrm{L})$ containing internal standard (0.05 wt.\% 3-(trimethylsilyl)propionic-2,2,3,3-d4 acid, sodium salt). Fourier Transform Infrared (FTIR) spectroscopy experiments were collected on a Perkin Elmer Spectrum One FT-IR Spectrometer (Waltham, MA, 
USA) in the spectral range of 400 to $4000 \mathrm{~cm}^{-1}$. Freeze-dried polymer and hydrogel samples (1-2 $\mathrm{mg}$ ) were pressed into $\mathrm{KBr}(200 \mathrm{mg})$ tablets.

\subsection{Synthesis of aldehyde-modified hyaluronan and alginate}

Aldehyde groups were generated from the vicinal diol groups of HA and AL by using periodate oxidation (Fig. 11(a), (b)) according to the previously reported method [18] that was slightly modified. Briefly, sodium hyaluronate or alginate $(0.500 \mathrm{~g})$ was dissolved in deionized water $(100 \mathrm{~mL})$. Sodium periodate (Table S1.) was dissolved in deionized water $(2.7 \mathrm{~mL})$, added dropwise and stirred for 2 hours to 4 hours (Table S1.) in the dark at room temperature under nitrogen. Ethylene glycol (4 equivalents) was added to inactivate any unreacted periodate and the solution was then stirred for 1 hour. Derivatized polymers were dialyzed with MW cutoff 25,000 membrane against deionized water for three days. Purified polymers were lyophilized to obtain white cotton-like products (HAALD and ALALD). ${ }^{1} \mathrm{H}$ NMR (Fig. 1. $\mathrm{D}_{2} \mathrm{O}, 300 \mathrm{MHz}$ ): HAALD: $\delta 4.47$ (m, 1H, H1 of glucose unit), 3.82-3.35 (m, 5H, H2-5 of glucose unit), 2.03 (s, $3 \mathrm{H},-\mathrm{NHC}(\mathrm{O}) \mathrm{CH}_{3}$ ). ALALD: 5.72 (s, 1H, H4-G), 5.26 and 5.18 (s, 1H, hemiacetal protons), 5.00 (s, 1H, H1-G), 4.59 (s, 1H, H1-M and H5-GM), 4.39 (s, 1H, H5-GG). FTIR (Fig. 2. KBr pellet): HAALD: $1720(v(\mathrm{C}=\mathrm{O})$ of $-\mathrm{C}(\mathrm{O}) \mathrm{H}), 1618(v(\mathrm{C}=\mathrm{O})$ of $-\mathrm{NHC}(\mathrm{O})-$ and $\mathrm{C}(\mathrm{O}) \mathrm{OH})$. ALALD: 1732 $(v(\mathrm{C}=\mathrm{O})$ of $-\mathrm{C}(\mathrm{O}) \mathrm{H})), 1634(v(\mathrm{C}=\mathrm{O})$ of $\mathrm{C}(\mathrm{O}) \mathrm{OH})$.

The DS\% of HAALD was determined using a TNBS method [16, 19]. Briefly, HAALD $(20 \mathrm{mg})$ was dissolved in acetate buffer $(2 \mathrm{~mL}, 0.1 \mathrm{M}, \mathrm{pH} 5.2)$ and added to TBC solution in acetate buffer ( $1 \mathrm{~mL}, 0.0348 \mathrm{~g}, 10$-fold excess per molar amount of sodium periodate used). The mixture was allowed to react for 1 hour at room temperature. $\mathrm{NaBH}_{3} \mathrm{CN}(1 \mathrm{~mL}, 0.0166$ $\mathrm{g}$, equimolar amount to TBC) in acetate buffer was added and allowed to react for 24 hours at room temperature under nitrogen. The polymer was dialyzed with MW cutoff 25,000 membrane against $0.1 \mathrm{M} \mathrm{NaCl}$ for 24 hours and for a further 24 hours in deionized water. Purified polymer was lyophilized to obtain a white cotton-like product. The ${ }^{1} \mathrm{H}$-NMR spectrum was measured and the DS\% was determined from the integration of the ${ }^{1} \mathrm{H}-\mathrm{NMR}$ peaks. ${ }^{1} \mathrm{H}$ NMR $\left(\mathrm{D}_{2} \mathrm{O}, 300\right.$ $\mathrm{MHz}): \delta 1.9\left(3 \mathrm{H}, \mathrm{NHCOCH}_{3}\right)$ and $1.3(9 \mathrm{H}, \mathrm{t}-\mathrm{Boc})$.

The DS\% of ALALD was determined using an aldehyde assay [19]. Briefly, aqueous ALALD solution $(0.5 \mathrm{~mL}, 10 \mathrm{mM})$ was mixed with $\mathrm{TBC}$ solution $(0.5 \mathrm{~mL}, 24.75 \mathrm{mM})$ in aqueous trichloroacetic acid $(1 \%)$ and allowed to react for 24 hours at room temperature. A portion of this solution $(200 \mu \mathrm{L})$ was mixed with aqueous TNBS solution $(2 \mathrm{~mL}, 4 \mathrm{mM}, 0.1 \mathrm{mM}$ borate buffer, $\mathrm{pH} 8$ ) and allowed to react for 30 minutes at room temperature. After this, it was diluted with aqueous hydrochloric acid $(0.5 \mathrm{M})$ and the absorbance was measured at $334 \mathrm{~nm}$. TBC was used as a standard to obtain a calibration curve. The blank sample was composed of deionized water $(0.5 \mathrm{~mL})$ and aqueous trichloroacetic acid $(0.5 \mathrm{~mL}, 1 \%)$.

\subsection{Synthesis of hydrazide-modified polyvinyl alcohol}

PVA was modified with hydrazide groups (Fig. 11(c)) according to the previously reported method [12]. Briefly, PVA (400 mg) was dissolved in dry DMSO $(8 \mathrm{~mL})$. CDI $(730 \mathrm{mg})$ was added under nitrogen at room temperature and the solution was mixed for 3 hours. Glysine ethyl ester hydrochloride $(126 \mathrm{mg})$ and triethylamine $(125 \mu \mathrm{L})$ were added and stirring was continued overnight under nitrogen. Finally, hydrazine $35 \mathrm{w}-\%$ aqueous solution $(8.6 \mathrm{~mL})$ was added. After 24 hours of stirring at room temperature, the solution was mixed with water (50 $\mathrm{mL}$ ) and reduced to about $8 \mathrm{~mL}$ of volume. The PVA derivative was precipitated from DMSO by using 10 -fold excess of mixture of diethyl ether/ethanol $(80 / 20 \mathrm{~mL})$. The precipitate was 
dissolved in a small amount of deionized water and dialyzed with MW cutoff 1000 membranes against deionized water for 3 days. Purified polymer was lyophilized to obtain a white cottonlike product (PVAHY). ${ }^{1} \mathrm{H}$ NMR (Fig. 1], $\left.\mathrm{D}_{2} \mathrm{O}, 300 \mathrm{MHz}\right): \delta 4.90(\mathrm{~m}, 1 \mathrm{H}$, polymer backbone $\mathrm{CH}$ of the modified unit), 4.00-3.72 (m, 3H, polymer backbone $\mathrm{CH}$ of the unmodified unit + $\left.\mathrm{CH}_{2} \mathrm{CONHNH}_{2}\right), 1.92-1.60\left(\mathrm{~m}, 2 \mathrm{H}\right.$, polymer backbone $\left.\mathrm{CH}_{2}\right)$. FTIR (Fig. 2, KBr pellet): 1710 $(v(\mathrm{C}=\mathrm{O})$ of $\mathrm{C}(\mathrm{O}) \mathrm{O}-), 1634(\mathrm{~d}(\mathrm{~N}-\mathrm{H})$ of prim. amine $), 1520(\mathrm{~d}(\mathrm{~N}-\mathrm{H})$ of sec. amine $), 1280(v(\mathrm{C}-\mathrm{O})$ of $\mathrm{C}(\mathrm{O}) \mathrm{O}-)$.

The DS\% of PVAHY was determined using a spectrophotometric method [12] based on the reaction between TNBS and the primary amines of PVAHY. Briefly, PVA $(0.8 \mathrm{mg})$ was dissolved in borate buffer $(2 \mathrm{~mL}, 0.1 \mathrm{M}, \mathrm{pH} 9.3)$. A sample $(0.1 \mathrm{~mL})$ was taken and diluted with borate buffer $(2.8 \mathrm{~mL})$. TNBS solution $(75 \mu \mathrm{L}, 0.025 \mathrm{M})$ was added and the solution was vigorously mixed. The mixture was allowed to stand for $30 \mathrm{~min}$ at room temperature to obtain a red color indicating primary amine groups. The absorbance was read at $505 \mathrm{~nm}$. A mixture of borate buffer $(2.9 \mathrm{~mL})$ and TNBS solution $(75 \mu \mathrm{L}, 0.025 \mathrm{M})$ was used as a blank reference. TBC was used as a standard to obtain a calibration curve.

\subsection{Formation of hydrogels}

Freeze-dried HAALD, ALALD and PVAHY components were dissolved in a $10 \%$ sucrose solution according to Table 1 . Sucrose was used to make the osmotic pressure more suitable for cells in the cell culture experiments. Equal volumes of components A and B were mixed together in a mold by using a dual syringe system to form HA-PVA and AL-PVA hydrogels (Table 1. Fig. 1.d)). Cut syringes were used as a mold to ease pushing the sample out.

The gelation time was determined using a tube tilt test [20], where the gel point is determined by tilting a tube containing the hydrogel solution. The gel point is the transition point from fluid to gel state, in other words, where the system stops flowing.

\subsection{Structural homogeneity}

The structural homogeneity of the hydrogels was studied using a dry to wet weight ratio test [21]. Three parallel cylinder shaped hydrogels were formed and slices of equal size were cut $(A-D$, diameter $\times$ height $=5 \mathrm{~mm} \times 5 \mathrm{~mm}$ ). The mass of the freshly made wet hydrogel slices $\left(\mathrm{W}_{\text {wet }}\right)$ was weighed prior to freeze-drying. The slices were weighed again after drying to obtain the mass of the dry hydrogel $\left(\mathrm{W}_{d r y}\right)$. The dry to wet ratios were calculated from the following:

$$
\text { dry to wet ratio }=\frac{W_{d r y}}{W_{w e t}}
$$

\subsection{Swelling kinetics}

The swelling kinetics of the hydrogels were studied in cell culture medium (DMEM/F12) and in deionized water. Three parallel, freshly made wet hydrogel samples $(100 \mu \mathrm{L})$ were weighed $\left(\mathrm{W}_{\text {initial }}\right)$ and placed into deionized water or cell culture medium and incubated at $37^{\circ} \mathrm{C}$. Samples were weighed at specific time points to obtain the mass of a swollen hydrogel $\left(\mathrm{W}_{\text {swollen }}\right)$. Swelling ratio (SR,\%) was calculated from the following:

$$
\operatorname{SR}(\%)=\frac{W_{\text {swollen }}-W_{\text {initial }}}{W_{\text {initial }}} \times 100
$$

Equilibrium was considered to have been achieved when the mass of the sample no longer changed but remained constant. 


\subsection{Enzymatic biodegradation}

The enzymatic degradation of hydrogels was studied using a hyaluronidase-enzyme (HAse). Three parallel hydrogel samples $(100 \mu \mathrm{L})$ were weighed, placed into HAse solution (20-50 $\mathrm{U} / \mathrm{mL}$, in PBS) and incubated at $37^{\circ} \mathrm{C}$ until the samples were completely degraded. The samples were weighed at different time points. The sample was considered to be degraded when there was visually no sample left to weigh. The residual mass (\%) was determined at each point and plotted as a mass loss curve.

\subsection{Mechanical properties of hydrogels and rabbit midbrain tissue}

The compression tests were performed using a BOSE Electroforce Biodynamic 5100 machine equipped with a $225 \mathrm{~N}$ load sensor and Wintest 4.1 software (Bose Corporation, Eden Prairie, Minnesota, USA). Five parallel hydrogel samples $(875 \mu \mathrm{L})$ were prepared into $5 \mathrm{~mL}$ cut syringes to ease pushing the sample out. The samples were left to stand for 24 hours to obtain uniform hydrogels. The surface of the platens was covered with Parafilm to avoid slipping of the sample. Samples were compressed using unconfined compression at a rate of $10 \mathrm{~mm} / \mathrm{min}$ to at least $65 \%$ to $70 \%$ strain. The measurements were conducted in the air at room temperature. The test data obtained from the measurement of New Zealand white rabbit midbrain samples (obtained from the animal experiments conducted at Tampere University Medical School, University of Tampere) were used as a reference [22].

The measuring data were imported to MS Excel, where stress and strain were plotted as graphs. The so-called stiffness of the material can be estimated based on the data and it was done similarly to Karvinen et al., 2017 [23]. Briefly, due to the characteristic non-linear elastic behavior of the hydrogels and tissues, the stress-strain curve cannot be described appropriately by Hooke's law (except at low strains). Therefore, the data were fitted to a 6th-order polynomial. Stiffness, $\tau$, can be defined as the derivative of stress $\sigma$ with respect to strain $\epsilon$. When the measured stress-strain data is represented by a polynomial, stiffness may be defined as follows:

$$
\frac{d \sigma}{d \epsilon} \equiv \tau(\epsilon)=\sum_{k=1}^{n} k c_{k} \epsilon^{k-1},
$$

where the coefficients of the polynomial $c_{k}$ are the so-called elastic constants [24, 25]. Here, $c_{1}$ is the second-order elastic constant, which is sometimes also called the elastic constant, Young's modulus or elastic stiffness constant [24, 25, 26]. The stiffness values obtained from the polynomial were plotted as a function of strain to be able to compare the behavior of materials at different strains. The second-order elastic constants were estimated based on the mean of stiffness polynomials of parallel samples at $0 \%$ to $15 \%$ strain with minimum standard deviations. It should be noted that the stiffness describes the same quantity as the second-order elastic constant, the latter being the stiffness at zero strain $\tau(0)=c_{1}$.

\subsection{Cell culture}

The cell culture experiments were performed using human pluripotent stem cell derived neuronal cells. Human embryonic stem cell (hESC) lines Regea08/023 and Regea11/013, and human induced pluripotent stem cell (iPCS) lines HEL 24.3 (kindly donated by Prof. Timo Otonkoski, University of Helsinki, Finland) and 04511.WT were used. BioMediTech has been granted ethical approval to derivate, culture and differentiate hESCs (Skottman, R05116) by the Pirkanmaa Hospital District. Moreover, permission has been granted by the National Authority for Medicolegal Affairs (FIMEA 1426/32/300/05) to conduct human stem cell research. Additionally, 
approval has been obtained to use hiPSC lines produced by other laboratories for neuronal research (R14023). The stem cell line derivation, culturing and characterization for all lines has been previously published [27, 28, 29]. The used stem cell lines are under constant quality control with frequent gene and protein expression analysis, karyotype and mycoplasma assays. Neuronal differentiation was performed using a suspension culture method [30]. Briefly, undifferentiated colonies were mechanically cut into small cell aggregates and transferred into the neural differentiation medium (NDM), containing 1:1 DMEM/F12: Neurobasal supplemented with GlutaMax (2 mM), 1 x B27, 1 x N2, penicillin/streptomycin $(25 \mathrm{U} / \mathrm{mL})$ and FGF2 $(20 \mathrm{ng} / \mathrm{mL})$. During the culture, cell aggregates formed round, freely floating neurospheres. During the differentiation, the neurospheres were kept small (diameter $\sim 500 \mu \mathrm{m}$ ) by cutting mechanically once a week. Half of the medium was changed three times a week.

The cells were plated as previously described [31, 22]. Briefly, pre-differentiated neuronal cell aggregates were mechanically cut into small cell clusters. These freshly cut small clusters were then plated either on top of the hydrogel surface or encapsulated inside the hydrogel. 2D growth controls were plated on top of mouse laminin $(10 \mu \mathrm{g} / \mathrm{mL})$ coated cell culture plastic. When culturing cells on top of the hydrogel surfaces, the gelation was performed directly into the cell culture well by combining equal volumes of components A and B similarly as described in Section 2.4 and mixed using a pipette tip. After complete gelation, the cells were plated on top of the hydrogel. When preparing 3D cultures with cells encapsulated inside the hydrogel, the cell clusters were mixed into PVAHY component just before performing the gelation. The cells were then cultured for two weeks either on top of or encapsulated inside the hydrogel. Cell culture media (NDM without FGF) was changed three times a week and cells were monitored during the culturing.

\subsection{Molecular biology analysis}

After two weeks in culture, the cells underwent viability analysis and immunocytochemical analysis as previously described [22]. Briefly, viability was analyzed with fluorescence-based LIVE/DEAD ${ }^{\circledR}$ viability/cytotoxicity assay (Thermo Fisher Scientific). The labels, Calcein-AM $(0.1 \mu \mathrm{M})$ that stains intact cells and ethidium homodimer-1 $(0.4 \mu \mathrm{M})$ that stains dead cells, were incubated for $30 \mathrm{~min}$ at $+37^{\circ} \mathrm{C}$ on the cells. After staining, the cells were imaged using an Olympus IX51 microscope (Olympus IX51 inverted microscope, PlanFLN 10×, and 20× objectives, Olympus DP30BW CCD camera). For immunocytochemistry, the cultures were fixed with $4 \%$ paraformaldehyde at room temperature for 30 minutes. After a brief wash in phosphate buffered saline (PBS), the cultures were incubated for 1 hour with blocking solution containing $10 \%$ normal donkey serum (NDS), $0.1 \%$ Triton X-100, and $1 \%$ bovine serum albumin (BSA) in PBS at room temperature, followed by another wash in $1 \%$ NDS, $0.1 \%$ Triton X-100 and $1 \%$ BSA in PBS. Then, the cultures were incubated with primary antibody solution in $1 \%$ NDS, $0.1 \%$ Triton $\mathrm{X}-100$, and $1 \% \mathrm{BSA}$ in PBS at $+4^{\circ} \mathrm{C}$ for 3 days. The antibodies used were MAP-2 (1:400) and B-tubulin (1:1000). After primary antibody incubation, the samples were washed three times in $1 \%$ BSA in PBS (the first time only briefly followed by $2 \times 1$ hour washes) and then incubated overnight at $+4^{\circ} \mathrm{C}$ with secondary antibodies (1:400). After secondary antibody incubation, the samples were again washed three times (the first time only briefly followed by 2 $\times 1$ hour washes) in PBS and then mounted with VECTASHIELD mounting media. The cultures were imaged using an Olympus IX51 microscope. 


\subsection{Statistical Data Analysis}

Statistical data analyses were performed with MATLAB (Statistics and Machine Learning Toolbox ${ }^{T M}$ ). All the quantitative data are presented as mean and standard deviation. A nonparametric Kruskal-Wallis test was used to determine whether there were statistically significant differences within the mechanical test data set. A Wilcoxon rank sum test was used to analyze the specific sample pairs for statistically significant differences. Non-parametric testing was chosen because the assumption of normally distributed data would be unjustifiable due to a relatively low $n$. When more than two groups were compared, the resulting p values were multiplied by the number of comparisons performed (Bonferroni correction). A p-value of $<0.05$ was considered significant.

\section{Results and Discussion}

\subsection{Modification of hyaluronan, alginate and polyvinyl alcohol with complementary reactive groups}

The polymers were modified with complementary reactive groups to attain aldehyde-modified HA (HAALD) and alginate (ALALD), and hydrazide-modified PVA (PVAHY) with relatively low DS\%, in order to not lose the characteristic properties of the polymers. Hyaluronan and alginate were periodate oxidized to obtain one ALALD component and four HAALD components with variable DS\% (Table 1). The modification was confirmed with ${ }^{1} \mathrm{H}-\mathrm{NMR}$ and FTIR analysis. In the ${ }^{1} \mathrm{H}-\mathrm{NMR}$ spectrum of HAALD (Fig. 1), the aldehyde peak (9 ppm to $10 \mathrm{ppm}$ ) was invisible. One explanation for this can be that, in water, aldehydes exist in equilibrium with their hydrated forms. They may also form reversible hemiacetals when reacting with some hydroxyl groups of HA [32]. In the FTIR spectrum (Fig. 2), the aldehyde shoulder $\left(1720 \mathrm{~cm}^{-1}\right)$ was observed. The signal partly overlaps with the carboxyl and amide $\mathrm{C}=\mathrm{O}$ stretching (1633 $\mathrm{cm}^{-1}$ ) signal, which makes observation more difficult. The success of ALALD modification was confirmed from the ${ }^{1} \mathrm{H}-\mathrm{NMR}$ spectrum (Fig. 1), where two new signals at $5.26 \mathrm{ppm}$ and 5.19 ppm can be found. Those correspond to a hemiacetalic proton that is formed when aldehyde reacts with the neighboring hydroxyl groups of alginate. In the FTIR spectrum of ALALD (Fig. 2), a new peak appeared at $1147 \mathrm{~cm}^{-1}$ (C-O-C), whereas the peaks at 879 and $818 \mathrm{~cm}^{-1}$ (C-O-C) weakened. The aldehyde shoulder at $1732 \mathrm{~cm}^{-1}$ was also found. Modifications were successful, although there are some things to be considered with regard to the reaction used. For example, periodate oxidation can lead to the loss of native backbone structure and a reduction in molecular weight, which might have an effect on the cells' ability to recognize HA molecule.

PVA was modified with hydrazide groups (PVAHY, DS\%=13). The ${ }^{1} \mathrm{H}-\mathrm{NMR}$ spectrum (Fig. 2) showed new $\mathrm{CH}$ peaks of the polymer backbone and the $\mathrm{CH}_{2}$ of the modified hydrazide unit around $4.90 \mathrm{ppm}$ and $4 \mathrm{ppm}$ to $3.72 \mathrm{ppm}$. In the FTIR spectrum (Fig. 2), the carbonyl $\mathrm{C}=\mathrm{O}$ and $\mathrm{C}-\mathrm{O}$ stretching signals $\left(1710\right.$ and $1280 \mathrm{~cm}^{-1}$ ) and the amine $\mathrm{N}-\mathrm{H}$ deformation signals (1634 and $1520 \mathrm{~cm}^{-1}$ ) of the modified unit were observed. There was also a $\mathrm{C}=\mathrm{O}$ stretching signal in the spectrum of the unmodified PVA, but this was due to the acetate groups left over from the manufacturing process.

\subsection{Formation of fast gelling hydrazone crosslinked hydrogels}

When choosing a fabrication method for a scaffold comprising living cells, biocompatibility should be considered. For example, extreme temperatures, toxic components, free radicals and organic solvents should all be avoided, whereas mild reaction conditions such as water-based 
methods are preferable [33, 34]. Injectable hydrogels can provide an alternative to physical implants due to their less invasive implantation process. An ideal implantation strategy would be to premix the cells with liquid gel precursors followed by injection and gelation in situ. To meet these requirements, hydrazone crosslinking was chosen as a fabrication method for our hydrogels.

Hydrazone crosslinked HA-PVA and AL-PVA hydrogels (Table 11 were prepared from modified polymer components by varying the DS\% and the molecular weight of the HA component and the polymer concentration of the hydrogel. HP1 and HP2 hydrogels contain a high molecular weight HAALD component, whereas HP3 and HP4 hydrogels contain a low molecular weight HAALD component. Additionally, the polymer concentration of the HP1 hydrogel was decreased, while keeping the other parameters within the group the same. Due to a higher water content and a lower number of crosslinkable groups, the decrease in the concentration led to softer hydrogels and a less constant shape. The other hydrogels had a stronger gel structure with better shape constancy that was presumably due to a higher crosslinking ratio. HP3 and HP4 hydrogels looked more crystalline and fragile when compared to HP1 and HP2 hydrogels. A stickiness to different surfaces such as plastic or glass, and a clear and transparent appearance were common for all the HA-PVA hydrogels. The AP hydrogel had a slightly yellowish appearance, but otherwise was similar to the HP1 and HP2 hydrogels. The presented hydrogels were chosen based on a preliminary study (data not shown), where it was shown, for example, that HA-PVA and AL-PVA hydrogels containing PVAHY component with lower DS\%, or ALALD component with higher DS\% did not gelate well.

The gelation time of hydrogels (Table 1) was determined using a tube tilt test (gives only estimated results). Mild chemoselective reaction produced soft hydrazone crosslinked HA-PVA and AL-PVA hydrogels with a gelation time ranging from seconds to minutes. The gelation time depended on the polymer concentration and the properties of the gel components. The gelation time of the HP1-based hydrogels increased from seconds to minutes when polymer concentration was decreased. The gelation time of the AP hydrogel was around 30 seconds. The gelation time of these hydrogels should be long enough for the proper mixing and injection of the cell-polymer solutions, although, it should be noted that injecting a large amount of fast-gelling material has a potential to damage the surrounding tissue. Injecting smaller amounts of gel over a longer period of time could be one option.

The chemical structure of the hydrogels was determined using a FTIR. The FTIR spectra (Fig. 2 showed the disappearance of an aldehyde signal (HAALD: 1720, ALALD: $1732 \mathrm{~cm}^{-1}$ ) and the appearance of a hydrazone $\mathrm{C}=\mathrm{N}$ stretching signal at $1635 \mathrm{~cm}^{-1}$ for the HA-PVA hydrogels and $1636 \mathrm{~cm}^{-1}$ for AP hydrogel. The change in the amine/amide signal (PVAHY: $1634 \mathrm{~cm}^{-1}$ ) is not easily detected because it overlaps with the hydrazone signal.

\subsection{Structural homogeneity}

In the ideal hydrogel, there exists a homogeneous distribution of crosslinks, but this is not the case in real hydrogels where spatial concentration fluctuations are common. This spatial gel inhomogeneity can negatively affect the hydrogel properties and functionality or cause uneven cell distribution if cells are involved. Commonly used methods to study the inhomogeneity are based on light scattering [35]. A simpler way to study the structural homogeneity is to determine the dry to wet ratios of the hydrogels. The dry to wet ratios of the hydrogels are presented in Fig. 3. The ratios were lower when the polymer concentration of the hydrogels was lowered. The HP1 hydrogel and the AP hydrogel showed the most linear (homogeneous) behavior between the slices. Hydrogels with higher DS\% (higher crosslinking density) and a 
lower molecular weight and polymer concentration showed more inhomogeneity. Based on the results, the inhomogeneity increased with the crosslinking density as was expected based on the literature [35]. This increase in inhomogeneity is caused by the simultaneous increase of the extent of network imperfections, which lead to regions with a different number of crosslinks.

\subsection{Swelling and deswelling kinetics}

The primary method for the estimation of hydrogel swelling rate is the quantifying of water content as a function of time. Therefore, swelling is not only a thermodynamic but also a kinetic process. The swelling ratio (SR, \%) of hydrogels is generally determined by studying either the water uptake of dry hydrogels or the water uptake of wet hydrogels which are dried after the swelling. The drawback of the first method is that the drying process can be harmful to the hydrogel structure and cause distortions to the swelling results. Moreover, these methods do not describe how initially wet hydrogel behaves, for example, during the cell culture experiment. For these reasons, the swelling kinetics of the wet hydrogels in deionized water and cell culture medium (DMEM/F12) were studied without drying the samples at any stage of the measurement. The SRs of the hydrogels are collected in Fig. 4. Results showed that in cell culture medium some hydrogels started to shrink in the presence of divalent ions. The HP1 and HP3 hydrogels (DS\%=5 HAALD) slightly swelled in cell culture medium, whereas other hydrogels shrank The lower the polymer concentrations of the hydrogel were, the more the hydrogels shrank. The SR was higher with hydrogels containing a lower molecular weight HAALD component when compared with similar gels containing a higher molecular weight HAALD component. The AP hydrogel shrank the most. Similar behavior was also observed during the cell culture experiments.

In deionized water, with no ions present, all the hydrogels swelled. The HA-PVA hydrogels containing a higher DS\% HAALD component (higher crosslinking) swelled less compared with the HA-PVA hydrogel containing a lower DS\% HAALD component. Moreover, a lower polymer concentration of the hydrogel led to a lower SR and to hydrogels that degraded faster during the experiment. Comparing hydrogels with similar polymer concentration and DS\% of the HAALD component, hydrogels with the lower molecular weight HAALD component swelled less. The swelling ratio for the AP hydrogel in water could not be determined because it was not stable in water.

The swelling was shown to be dependent on the solvent and its ionic strength (Fig 4). In cell culture medium, most hydrogels did not swell, but instead they shrank. This was expected because in the presence of salts hydrogel networks lose their hydrophilic-hydrophobic balance and shrink due to ex-osmosis. Counter ions $\left(\mathrm{Na}^{+}\right)$condense around the fixed carboxylate ion charges of hyaluronan or alginate and cause a decrease in repulsive forces among the carboxylate groups. This leads to a decrease in swelling. The cell culture medium and body fluids also contain divalent cations. Therefore, it can be expected that when placed into such media or fluids, the AP hydrogel may also form ionic crosslinks leading to a more crosslinked structure and swelling will be reduced, as was also reported by Kuo and Ma, 2008 [36]. The adsorption of water was many times that of cell culture medium. The degree to which the volume increases depends on the degree of crosslinking and the degree to which the polymer chains like to have solvent molecules around them. Due to the many hydrophilic groups of HA-PVA hydrogels, they swelled more in water. The more hydrophilic the structure, the stronger the polymer-water interaction becomes [37]. The swelling ratio will be lower when the degree of crosslinking is higher due to the tighter gel structure and less mobile polymer chains. This was seen with the HA-PVA hydrogels. 


\subsection{Enzymatically biodegradable hyaluronan-based hydrogels}

In biological systems, hyaluronan is degraded by hyaluronidase (HAse) enzyme, which cleaves the $\beta-1,4-$ glycosidic linkage. Hyaluronan itself is degraded sufficiently fast, whereas crosslinking makes it more resistant to degradation. Here, the enzymatic biodegradation of the HA-PVA hydrogels was determined using a higher amount of HAse $(20-50 \mathrm{U} / \mathrm{mL})$ than in physiological conditions, and the degradation was followed by monitoring the bulk mass loss. The degradation curves are shown in Fig. 5. It was notable that almost all the gels swelled first before any mass loss could be detected. The hydrogels were completely degraded in 21 to 25 hours. Degradation was faster when the polymer concentration of the hydrogels decreased, and when both the molecular weight and DS\% of the HAALD component were lower. Higher crosslinking density delayed the degradation. Overall, the results showed that HAse can recognize the crosslinked oxidized hyaluronan, which indicates that these hydrogels could also be degraded in vivo, as has also been shown in the literature [16, 32, 38, 39, 40]. Degradation products, rather than the polymer itself, may have a critical influence on the biocompatibility. Degradation products of HA, N-acetylglucosamine at the reducing terminus and glucuronic acid at the nonreducing end, are cleared from the body, but they have also been found to modulate wound healing [41]. Here, only the degradation of HA-PVA hydrogels was studied. Alginate is inherently nondegradable in mammals because they lack the enzyme (i.e., alginase) that can cleave the polymer chains. However, it is said that alginate can be made degradable in physiological conditions by partial oxidation [42], which was also used in our study.

\subsection{Brain mimicking mechanical properties of hydrogels}

Stiffness is a constant over the range of strain for many materials. Such materials are called linear. For tissues and hydrogels, the stress-strain curve is non-linear in the elastic portion, even at small strains. Therefore, a polynomial fit was used for the data and the stiffness of materials was determined similar to Karvinen et al., 2017 [23]. Moreover, instead of giving only the second-order elastic constants for the materials, the stiffness as a function of strain was shown in order to represent the material behavior in a wider strain range. The representative compressive stress as a function of the deformation strain curves, and the stiffness as a function of the strain curves of the HA-PVA and AL-PVA hydrogels and midbrain tissue are shown in Fig. 6.

The deformation of the hydrogel depends on the structure of the hydrogel. The shape of the stress-strain curves (Fig. 6(a)) showed that when the stress was increased, the polymer chains in the structure re-orientate at the beginning before the hydrogels start to resist the applied force. All the hydrogels were initially resistant to deformation and became progressively stiffer when the load was increased leading finally to the fracture of the hydrogels. The HP2 and AP hydrogels had the lowest fracture strains (50\% to 57\%) compared with the others (over 60\%). The stressstrain curves of the hydrogels behaved similarly to midbrain tissue at lower strains, but at higher strains the curve of the midbrain tissue became steeper showing no clear fracture. In addition, it was noted that at lower strain values, HP4 hydrogel was stiffer than midbrain tissue, but at higher strains (over 20\% strain) the opposite was the case. This would mean that for midbrain tissue a greater force is required to fracture it. Altogether, the stress-strain curves of the brain tissue and the hydrogels were in the same range at lower strains and showed similar elastic behavior, whereas at higher strains in the fracture area the difference was clearer.

Stiffness was also plotted as a function of strain. The stiffness-strain curves (Fig. 6 (b)) showed that the hydrogels were initially resistant to deformation, but became progressively stiffer with increasing strain (also known as strain hardening [43]) indicating a non-linear deformation 
characteristic of hydrogels under compression. At certain strain the stiffness started to decrease, which indicated some irreversible changes in the hydrogel structure. When the drop happens sooner, it indicates that the material can withstand less deformation before the final fracture caused by a permanent deformation. Hydrogels with higher fracture strain are considered to be more elastic. The stiffness-strain curves showed that the stiffness was strain dependent and quite constant at around $20 \%$ strain with all the hydrogels. After that, the stiffness increased more or less depending on the sample and at a specific point started to drop. This drop occured sooner for the HP2 and AP hydrogels when compared with the others. The stiffness decreased when the polymer concentration of the hydrogel decreased. At low strain values, hydrogels containing a low molecular weight HAALD component or a higher DS\% had higher stiffness. In addition, the stiffness of the AP hydrogel was similar to the lowest HP1b hydrogel. At lower strain values, the stiffness of the HP1 hydrogel was the most similar to midbrain tissue.

The second-order elastic constants for the hydrogels and midbrain tissue are presented in Fig. 6(c). The HP1b hydrogel showed a statistically significant difference $p<0.01$ with respect to the HP1, HP1a, HP2, HP3 and HP4 hydrogels. The HP4 hydrogel showed significant difference p< 0.05 with respect to the HP1a, HP3 and AP hydrogels and midbrain, and $\mathrm{p}<0.01$ with respect to the HP1 and HP1b hydrogels. Whereas, the AP hydrogel showed significant difference $\mathrm{p}<0.05$ with respect to the HP1, HP2, HP3 and HP4 hydrogels. No statistically significant differences were detected between other materials. Overall, the second-order elastic constants of hydrogels were relatively low $(0.9 \mathrm{kPa}$ to $5.1 \mathrm{kPa})$ and similar to midbrain tissue, which could indicate their suitability for neural application. In particular, the HP1 hydrogel showed the most similar elastic behavior (and second-order elastic constant) compared with midbrain tissue at lower strains. The second-order elastic constant of brain tissue was in the same range as reported in previous studies [10, 44]. This was true, even though the same data of brain tissue had been previously analyzed by Koivisto et al., 2017 [22] showing that the constant, or as they call it, the compressive modulus was slightly higher, and thus deviated from the literature.

In this study, the bulk properties of hydrogels were tested. In biological tissues, there are local regions with variable stiffness due to the composite character and ongoing remodeling of the ECM [45, 46]. Because cells respond to these spatial variations, the local mechanical properties should be crucial for hydrogel design and should also be measured, for example, with atomic-force microscopy (AFM) [46]. The drawback of this method is that it only measures the surface of the material (2D), but does not really tell anything about the 3D environment.

\subsection{High molecular weight HA-PVA hydrogels and AL-PVA hydrogels supported human neu- ronal cells}

Hydrogel compositions were first tested as a growth surface for the cells. The human neuronal cells were cultured on top of the hydrogel surfaces and analyzed after two weeks of culturing. All tested hydrogel compositions supported cell growth and no cytotoxicity was observed (data not shown). The best compositions were the HA-PVA hydrogels containing a high molecular weight HAALD component (HP1, HP2) as well as the AP hydrogel. These both supported cell growth and neurite outgrowth along the hydrogel surface (Fig. 7 (a), (c), (e)), whereas the HP3 and HP4 hydrogels containing a low molecular weight HAALD component were less-supportive (Fig. 7 (b), (d)). The molecular weight of HA is known to alter cell behavior, such as adhesion and growth, in many cell types and tissues reviewed by Cyphert et al., 2015[47]. Especially in neural tissue, the high molecular weight HA is linked to decreasing glial scar formation [48]. Neurite outgrowth from cell aggregates was the highest in the HP1 hydrogel. The increase of 
the DS\% of the HAALD component in HA-PVA hydrogels had a negative effect on neurite outgrowth when the HP1 and HP2 hydrogels were compared (Fig. 7 $7 \mathrm{f}$ )). The effect of increasing the DS\% was not as dramatic on the cells as the change in molecular weight. This might be due to the mechanisms of HA recognition in the cell membrane [47]. The most prominent compositions, HP1 and AL, had the lowest stiffness of all the tested hydrogels. Overall, material stiffness (mechanical environment) is known to affect neural cell viability and behavior by directing neural cell lineage, proliferation and growth [4, 46]. Moreover, cultured cells have been shown to better retain their functional phenotype when cultured on material with a similar stiffness to native ECM [49]. Our findings are in line with previously published studies that suggest softer materials are more favored by the neural cells [50, 4].

\subsection{Human neuronal cells preferred low stiffness hydrogels}

The best hydrogel compositions, HP1 and AP, were selected for further 3D culturing experiments. Here, the cells were cultured encapsulated in the hydrogel. First, the viability of neuronal cells inside the HP1 and AP hydrogels was analyzed after two weeks in culture. The cell viability in 3D was at a similar level as viability in 2D control cultures on top of laminin (Fig. 8 (a-c)). This indicates that the hydrogel was well tolerated as a culturing environment. Next, the effect of the decreased polymer concentration of the HP1 hydrogel was studied in order to see how the cells behaved in even softer hydrogels. Neuronal cells were cultured for two weeks encapsulated inside the four soft hydrogel compositions (HP1, HP1a, HP1b and AP). Cell growth was seen inside all the studied compositions and the cells were positive for the neuronal markers MAP-2 and B-tubulin III (Fig. 8 $(\mathrm{d}-\mathrm{g})$ ). The stiffness of the material has been previously reported to be related to cell type: neurons prefer lower compressive modulus $(0.1 \mathrm{kPa}$ to $1 \mathrm{kPa})$, whereas astrocytes and oligodendrocytes prefer slightly higher $(0.5 \mathrm{kPa}$ to $10 \mathrm{kPa})$ [4]. In our studies with human neuronal cells, the cultures contained a high number of neurons even though the mechanical properties of the hydrogels used in this study are reported to favor a mixed population of neurons and glial cells or rather glial fate [4]. One explanation for the different result might be in the different hydrogels used in the studies. Slight differences were seen in the cell growth, for example, in the HP1 and HP1a hydrogels more cells stayed in clusters (Fig. 8 (d), (e)), whereas inside the softest HP1b and AP hydrogels the cell outgrowth from the aggregates into the hydrogel was more robust (Fig. 8(f), (g)). From our results with HA-based hydrogels, we can conclude that lowering the polymer concentration of the hydrogel had a positive effect on neuronal growth. Lower concentration hydrogels were softer and had a lower crosslinking density leaving more space inside the hydrogel. Nevertheless, it is not known how these changes caused the positive effect on cell growth.

\section{Conclusions}

In summary, injectable hydrazone crosslinked HA-PVA and AL-PVA hydrogels were produced and their detailed properties were investigated. To the best of our knowledge, the polymerization and properties of hydrazone crosslinked AL-PVA hydrogel are reported for the first time. The degree of substitution and molecular weight of the polymer components as well as the polymer concentration of the hydrogel were shown to affect to the swelling, degradation and mechanical properties of the hydrogels. Furthermore, the suitability of these hydrogels to be used as a supportive biomaterial for 3D neuronal cell culture was studied. The effect of the above parameters on the growth of human pluripotent stem cell-derived neuronal cells was studied. The most 
supportive HA-PVA and AL-PVA hydrogels for neural cells had brain-mimicking mechanical properties and were composed of a high molecular weight HA component. It was also shown that lowering the polymer concentration of hydrogel enhanced the neuronal growth. In this study, it was shown that neuronal spreading and 3D neural network formation were enhanced inside the softest hydrogels.

\section{Acknowledgements}

This work was funded by TEKES (the Finnish Funding Agency for Innovation) Human Spare Parts project, the Finnish Cultural Foundation grant numbers 00140325 and 00150312 , and by the Academy of Finland grant number 286990. The authors would like to thank Ph.D. Mari Hämäläinen (University of Tampere Medical School) for providing the rabbit tissue samples. The authors would also like to thank Ph.D. Alexandre Efimov and Laboratory Attendant AnneMaarit Tikkanen (Faculty of Natural Sciences, Laboratory of Chemistry and Bioengineering, Tampere University of Technology, Tampere, Finland) for their help related to the NMR- and FTIR-measurements.

\section{Appendix A. Supplementary material}

\section{References}

[1] X. Wang, J. He, Y. Wang, F.-Z. Cui, Hyaluronic acid-based scaffold for central neural tissue engineering, Interface focus (2012) rsfs20120016.

[2] A. R. Murphy, A. Laslett, C. M. O'Brien, N. R. Cameron, Scaffolds for 3d in vitro culture of neural lineage cells, Acta Biomaterialia 54 (2017) 1-20.

[3] C. Cha, S. Y. Kim, L. Cao, H. Kong, Decoupled control of stiffness and permeability with a cell-encapsulating poly (ethylene glycol) dimethacrylate hydrogel, Biomaterials 31 (18) (2010) 4864-4871.

[4] E. R. Aurand, K. J. Lampe, K. B. Bjugstad, Defining and designing polymers and hydrogels for neural tissue engineering, Neuroscience research 72 (3) (2012) 199-213.

[5] D. R. Nisbet, K. E. Crompton, M. K. Horne, D. I. Finkelstein, J. S. Forsythe, Neural tissue engineering of the CNS using hydrogels, Journal of Biomedical Materials Research Part B: Applied Biomaterials 87 (1) (2008) 251-263.

[6] S. Wu, R. Xu, B. Duan, P. Jiang, Three-dimensional hyaluronic acid hydrogel-based models for in vitro human ipsc-derived npc culture and differentiation, Journal of Materials Chemistry B 5 (2017) 3870-3878.

[7] M. M. Adil, T. Vazin, B. Ananthanarayanan, G. M. Rodrigues, A. T. Rao, R. U. Kulkarni, E. W. Miller, S. Kumar, D. V. Schaffer, Engineered hydrogels increase the post-transplantation survival of encapsulated hesc-derived midbrain dopaminergic neurons, Biomaterials 136 (2017) 1-11.

[8] Z. Wei, J. Zhao, Y. M. Chen, P. Zhang, Q. Zhang, Self-healing polysaccharide-based hydrogels as injectable carriers for neural stem cells, Scientific reports 6 (2016) 37841.

[9] G. Palazzolo, N. Broguiere, O. Cenciarelli, H. Dermutz, M. Zenobi-Wong, Ultrasoft alginate hydrogels support long-term three-dimensional functional neuronal networks, Tissue Engineering Part A 21 (15-16) (2015) 21772185 .

[10] S. K. Seidlits, Z. Z. Khaing, R. R. Petersen, J. D. Nickels, J. E. Vanscoy, J. B. Shear, C. E. Schmidt, The effects of hyaluronic acid hydrogels with tunable mechanical properties on neural progenitor cell differentiation, Biomaterials 31 (14) (2010) 3930-3940.

[11] A. D. Augst, H. J. Kong, D. J. Mooney, Alginate hydrogels as biomaterials, Macromolecular bioscience 6 (8) (2006) 623-633.

[12] D. A. Ossipov, K. Brännvall, K. Forsberg-Nilsson, J. Hilborn, Formation of the first injectable poly (vinyl alcohol) hydrogel by mixing of functional pva precursors, Journal of applied polymer science 106 (1) (2007) 60-70.

[13] R. H. Schmedlen, K. S. Masters, J. L. West, Photocrosslinkable polyvinyl alcohol hydrogels that can be modified with cell adhesion peptides for use in tissue engineering, Biomaterials 23 (22) (2002) 4325-4332.

[14] J. M. Ino, P. Chevallier, D. Letourneur, D. Mantovani, C. Le Visage, Plasma functionalization of poly (vinyl alcohol) hydrogel for cell adhesion enhancement, Biomatter 3 (4) (2013) e25414.

[15] Y. Jiang, J. Chen, C. Deng, E. J. Suuronen, Z. Zhong, Click hydrogels, microgels and nanogels: emerging platforms for drug delivery and tissue engineering, Biomaterials 35 (18) (2014) 4969-4985. 
[16] D. A. Ossipov, S. Piskounova, J. Hilborn, Poly (vinyl alcohol) cross-linkers for in vivo injectable hydrogels, Macromolecules 41 (11) (2008) 3971-3982.

[17] C. Aulin, K. Bergman, M. Jensen-Waern, P. Hedenqvist, J. Hilborn, T. Engstrand, In situ cross-linkable hyaluronan hydrogel enhances chondrogenesis, Journal of tissue engineering and regenerative medicine 5 (8) (2011) e188e196.

[18] X. Jia, Y. Yeo, R. J. Clifton, T. Jiao, D. S. Kohane, J. B. Kobler, S. M. Zeitels, R. Langer, Hyaluronic acid-based microgels and microgel networks for vocal fold regeneration, Biomacromolecules 7 (12) (2006) 3336-3344.

[19] K. H. Bouhadir, D. S. Hausman, D. J. Mooney, Synthesis of cross-linked poly (aldehyde guluronate) hydrogels, Polymer 40 (12) (1999) 3575-3584.

[20] ASTM Standard F2900:2011, Standard Guide for Characterization of Hydrogels used in Regenerative Medicine, ASTM International, West Conshohocken, PA, USA, 2011.

[21] C. K. Kuo, P. X. Ma, Ionically crosslinked alginate hydrogels as scaffolds for tissue engineering: part 1. structure, gelation rate and mechanical properties, Biomaterials 22 (6) (2001) 511-521.

[22] J. Koivisto, T. Joki, J. Parraga, R. Pääkkönen, L. Ylä-Outinen, L. Salonen, I. Jonkkari, M. Peltola, T. Ihalainen, S. Narkilahti, et al., Bioamine-crosslinked gellan gum hydrogel for neural tissue engineering, Biomedical materials 12 (2) (2017) 025014.

[23] J. Karvinen, J. T. Koivisto, I. Jönkkäri, M. Kellomäki, The production of injectable hydrazone crosslinked gellan gum-hyaluronan-hydrogels with tunable mechanical and physical properties, Journal of the Mechanical Behavior of Biomedical Materials 71 (2017) 383-391.

[24] K. Brugger, Thermodynamic definition of higher order elastic coefficients, Physical Review 133 (6A) (1964) A1611.

[25] J. F. Nye, Physical properties of crystals: their representation by tensors and matrices, Oxford university press, 1985.

[26] W. D. Callister, D. G. Rethwisch, Materials science and engineering: an introduction, Vol. 7, Wiley New York, 2007.

[27] M. Ojala, C. Prajapati, R.-P. Pölönen, K. Rajala, M. Pekkanen-Mattila, J. Rasku, K. Larsson, K. Aalto-Setälä, Mutation-specific phenotypes in hipsc-derived cardiomyocytes carrying either myosin-binding protein $\mathrm{C}$ or $\alpha$ tropomyosin mutation for hypertrophic cardiomyopathy, Stem cells international 2016 (ID 1684792) (2016) 1-16.

[28] K. Rajala, H. Hakala, S. Panula, S. Aivio, H. Pihlajamäki, R. Suuronen, O. Hovatta, H. Skottman, Testing of nine different xeno-free culture media for human embryonic stem cell cultures, Human Reproduction 22 (5) (2007) $1231-1238$.

[29] S. Toivonen, M. Ojala, A. Hyysalo, T. Ilmarinen, K. Rajala, M. Pekkanen-Mattila, R. Äänismaa, K. Lundin, J. Palgi, J. Weltner, et al., Comparative analysis of targeted differentiation of human induced pluripotent stem cells (hiPSCs) and human embryonic stem cells reveals variability associated with incomplete transgene silencing in retrovirally derived hiPSC lines, Stem cells translational medicine 2 (2) (2013) 83-93.

[30] R. S. Lappalainen, M. Salomäki, L. Ylä-Outinen, T. J. Heikkilä, J. A. Hyttinen, H. Pihlajamäki, R. Suuronen, H. Skottman, S. Narkilahti, Similarly derived and cultured hESC lines show variation in their developmental potential towards neuronal cells in long-term culture, Regenerative medicine 5 (5) (2010) 749-762.

[31] L. Ylä-Outinen, T. Joki, M. Varjola, H. Skottman, S. Narkilahti, Three-dimensional growth matrix for human embryonic stem cell-derived neuronal cells, Journal of tissue engineering and regenerative medicine 8 (3) (2014) 186-194.

[32] D. A. Ossipov, S. Piskounova, O. P. Varghese, J. Hilborn, Functionalization of hyaluronic acid with chemoselective groups via a disulfide-based protection strategy for in situ formation of mechanically stable hydrogels, Biomacromolecules 11 (9) (2010) 2247-2254.

[33] H.-W. Chien, W.-B. Tsai, S. Jiang, Direct cell encapsulation in biodegradable and functionalizable carboxybetaine hydrogels, Biomaterials 33 (23) (2012) 5706-5712.

[34] M. C. LaPlaca, V. N. Vernekar, J. T. Shoemaker, D. K. Cullen, Three-dimensional neuronal cultures, Methods in bioengineering: 3D tissue engineering. Norwood, MA: Artech House (2010) 187-204.

[35] G. Gerlach, K.-F. Arndt, Hydrogel sensors and actuators: engineering and technology, Vol. 6, Springer Science \& Business Media, 2009.

[36] C. K. Kuo, P. X. Ma, Maintaining dimensions and mechanical properties of ionically crosslinked alginate hydrogel scaffolds in vitro, Journal of Biomedical Materials Research Part A 84 (4) (2008) 899-907.

[37] N. A. Peppas, R. M. Ottenbrite, K. Park, T. Okano, Biomedical applications of hydrogels handbook, Springer Science \& Business Media, 2010.

[38] M. Patenaude, T. Hoare, Injectable, mixed natural-synthetic polymer hydrogels with modular properties, Biomacromolecules 13 (2) (2012) 369-378.

[39] J. Kim, I. S. Kim, T. H. Cho, K. B. Lee, S. J. Hwang, G. Tae, I. Noh, S. H. Lee, Y. Park, K. Sun, Bone regeneration using hyaluronic acid-based hydrogel with bone morphogenic protein-2 and human mesenchymal stem cells, Biomaterials 28 (10) (2007) 1830-1837. 
[40] R. Stern, G. Kogan, M. J. Jedrzejas, L. Šoltés, The many ways to cleave hyaluronan, Biotechnology advances 25 (6) (2007) 537-557.

[41] A. Fakhari, C. Berkland, Applications and emerging trends of hyaluronic acid in tissue engineering, as a dermal filler and in osteoarthritis treatment, Acta biomaterialia 9 (7) (2013) 7081-7092.

[42] K. Y. Lee, D. J. Mooney, Alginate: properties and biomedical applications, Progress in polymer science 37 (1) (2012) 106-126.

[43] G. Lamouche, B. F. Kennedy, K. M. Kennedy, C.-E. Bisaillon, A. Curatolo, G. Campbell, V. Pazos, D. D. Sampson, Review of tissue simulating phantoms with controllable optical, mechanical and structural properties for use in optical coherence tomography, Biomedical optics express 3 (6) (2012) 1381-1398.

[44] K. J. Lampe, R. G. Mooney, K. B. Bjugstad, M. J. Mahoney, Effect of macromer weight percent on neural cell growth in 2D and 3D nondegradable PEG hydrogel culture, Journal of Biomedical Materials Research Part A 94 (4) (2010) 1162-1171.

[45] J. Y. Wong, J. B. Leach, X. Q. Brown, Balance of chemistry, topography, and mechanics at the cell-biomateria interface: Issues and challenges for assessing the role of substrate mechanics on cell response, Surface Science 570 (1) (2004) 119-133.

[46] F. Brandl, F. Sommer, A. Goepferich, Rational design of hydrogels for tissue engineering: impact of physical factors on cell behavior, Biomaterials 28 (2) (2007) 134-146.

[47] J. M. Cyphert, C. S. Trempus, S. Garantziotis, Size matters: molecular weight specificity of hyaluronan effects in cell biology, International journal of cell biology 2015 (2015) 1-8.

[48] S. Hou, Q. Xu, W. Tian, F. Cui, Q. Cai, J. Ma, I.-S. Lee, The repair of brain lesion by implantation of hyaluronic acid hydrogels modified with laminin, Journal of neuroscience methods 148 (1) (2005) 60-70.

[49] W. L. K. Chen, C. A. Simmons, Lessons from (patho) physiological tissue stiffness and their implications for drug screening, drug delivery and regenerative medicine, Advanced drug delivery reviews 63 (4) (2011) 269-276.

[50] L. A. Flanagan, Y.-E. Ju, B. Marg, M. Osterfield, P. A. Janmey, Neurite branching on deformable substrates, Neuroreport 13 (18) (2002) 2411 


\begin{tabular}{l|ccc|ccc|c|c}
\hline $\begin{array}{l}\text { Gel } \\
\text { code }\end{array}$ & Component A & DS \% & $\begin{array}{c}\text { Concentration } \\
\mathrm{mg} / \mathrm{mL}\end{array}$ & Component B & DS \% & $\begin{array}{c}\text { Concentration } \\
\mathrm{mg} / \mathrm{mL}\end{array}$ & $\begin{array}{c}\text { Polymer } \\
\text { conc. \% }\end{array}$ & $\begin{array}{c}\text { Gelation } \\
\text { time }\end{array}$ \\
\hline HP1 & HAALD1H & 5 & 20 & PVAHY & 13 & 10 & 1.5 & seconds \\
HP1a & HAALD1H & 5 & 10 & PVAHY & 13 & 5 & 0.75 & $1-5$ min \\
HP1b & HAALD1H & 5 & 5 & PVAHY & 13 & 2.5 & 0.375 & 5 min \\
HP2 & HAALD2H & 9 & 25 & PVAHY & 13 & 5 & 1.5 & seconds \\
HP3 & HAALD1L & 5 & 20 & PVAHY & 13 & 10 & 1.5 & seconds \\
HP4 & HAALD2L & 9 & 20 & PVAHY & 13 & 10 & 1.5 & seconds \\
AP & ALALD & 7 & 20 & PVAHY & 13 & 10 & 1.5 & 30 seconds \\
\hline
\end{tabular}

Table 1: Compositions and gelation time of hydrazone crosslinked HA-PVA and AL-PVA hydrogels. At the end of the name of component A, $\mathrm{H}$ denotes high $\mathrm{M}_{w}\left(1.5-1.8 \times 10^{6}\right.$ $\mathrm{g} / \mathrm{mol})$ and $\mathrm{L}$ denotes low $\mathrm{M}_{w}\left(1.5 \times 10^{5} \mathrm{~g} / \mathrm{mol}\right)$. 
(a)

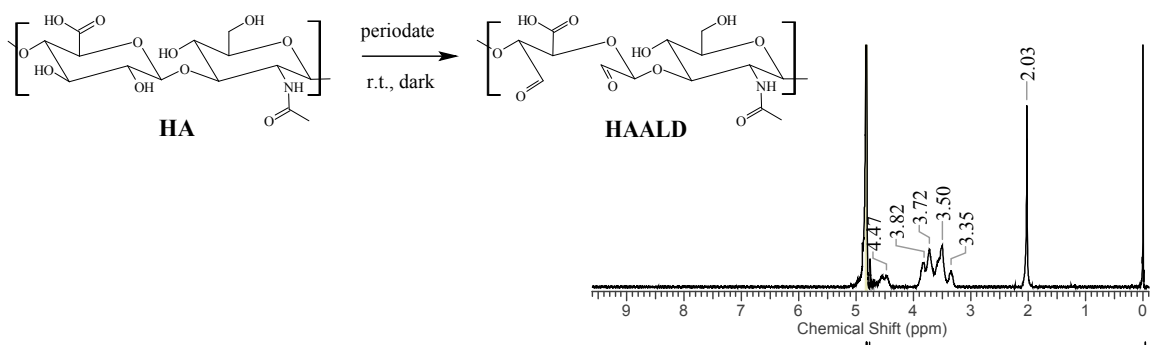

(b)

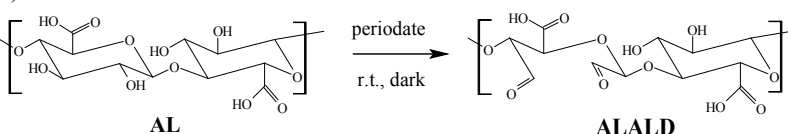

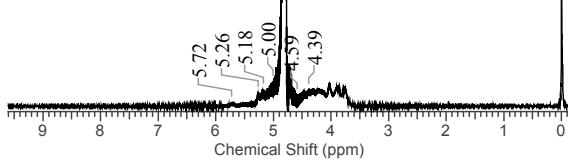

(c)

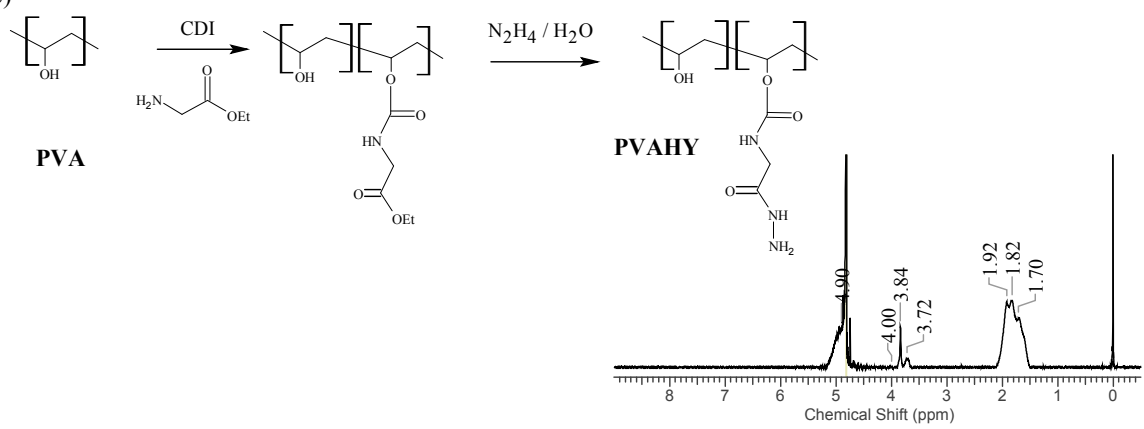

(d)
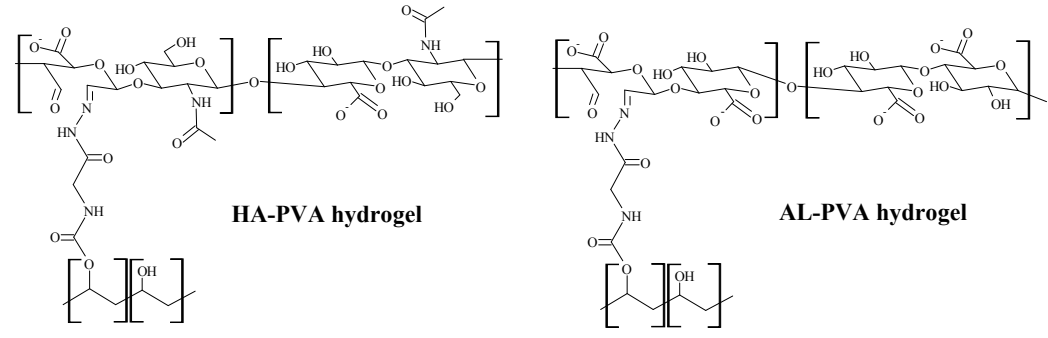

Figure 1: Reaction schemes of the oxidation of (a) HA (HAALD) and (b) AL (ALALD), and (c) the hydrazidemodification of PVA (PVAHY). The ${ }^{1} \mathrm{H}-\mathrm{NMR}$ spectra of hydrogel components are presented next to the structures. (d) The chemical structures of HA-PVA and AL-PVA hydrogels formed by hydrazone crosslinking. 
(a)
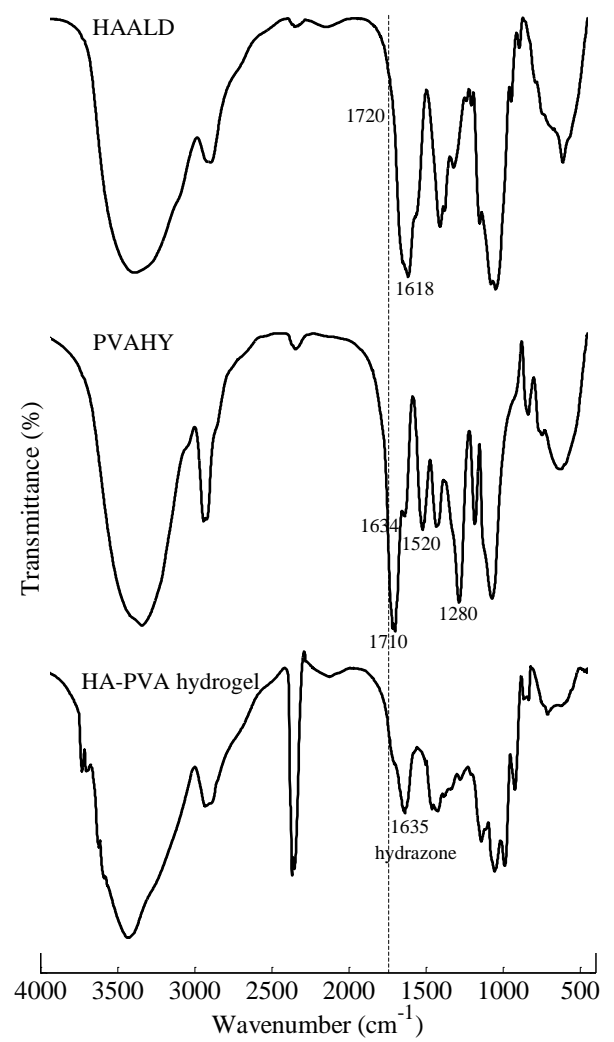

(b)
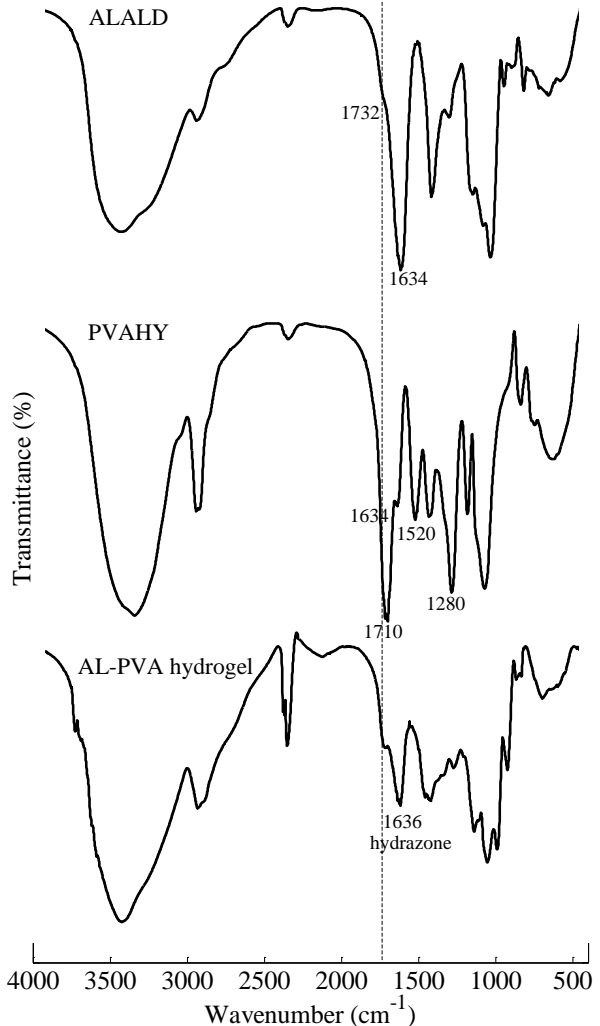

Figure 2: FTIR spectra of (a) HA-PVA, and (b) AL-PVA hydrogels, including their components. The peak of $2300 \mathrm{~cm}^{-1}$ is caused by the $\mathrm{CO}_{2}$ problem of the Spectrometer and is not related to the samples. 


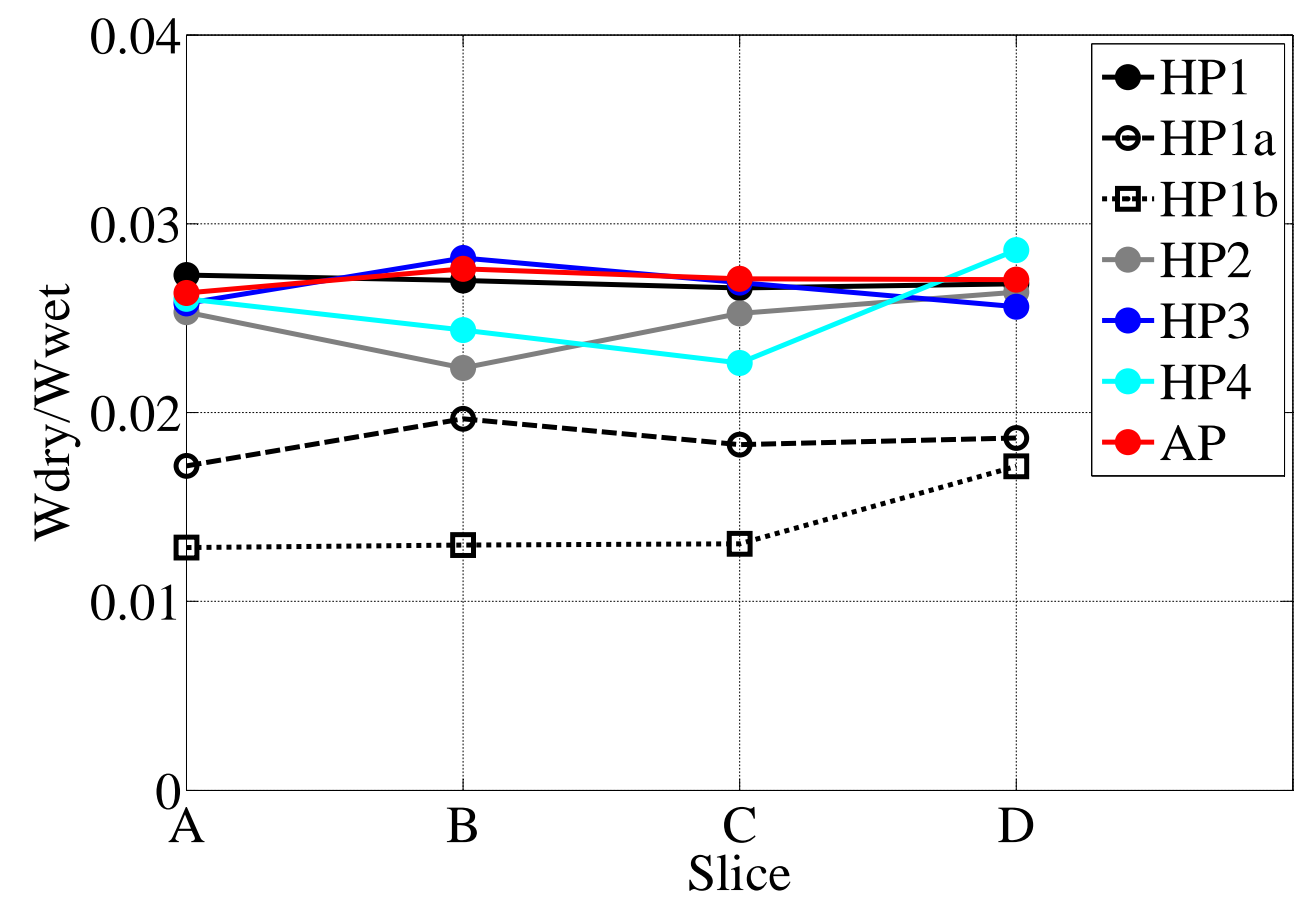

Figure 3: Homogeneity of the hydrogels based on the calculated average values of dry to wet ratios $(n=3)$. Homogeneity is connected to the linearity of dry to wet ratios between different slices (A to D) of the same gel sample. 
(a)

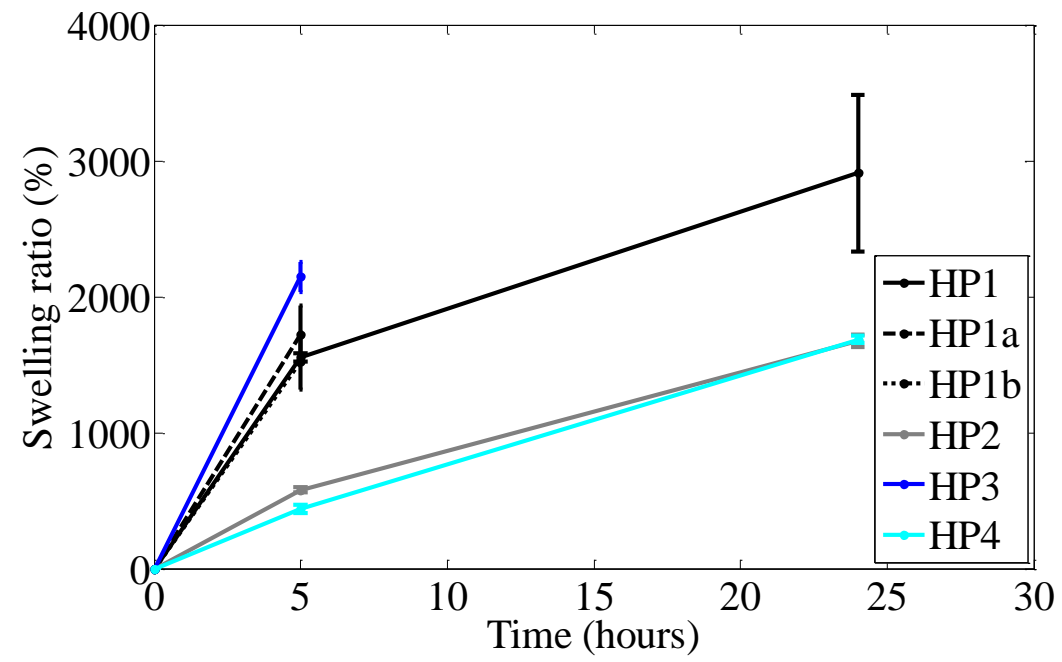

(b)

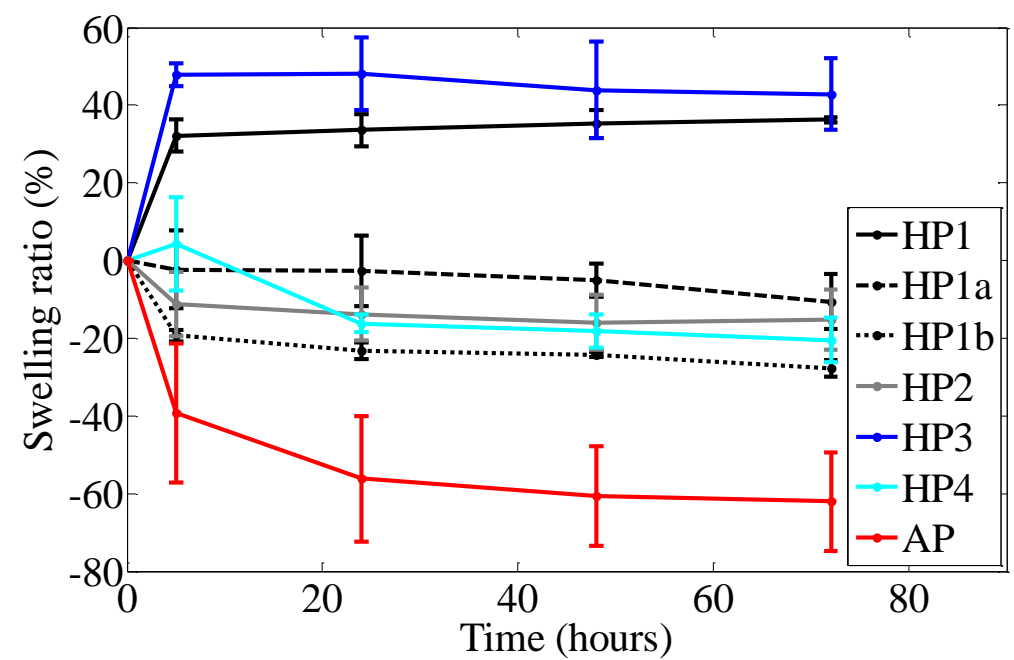

Figure 4: Swelling ratios (SR,\%) of the initially wet hydrogels in (a) deionized water and in (b) cell culture medium (DMEM/F12). AP hydrogel was not stable in water and degraded in a few hours. The mean $(n=3)$ and standard deviation bars are shown. 


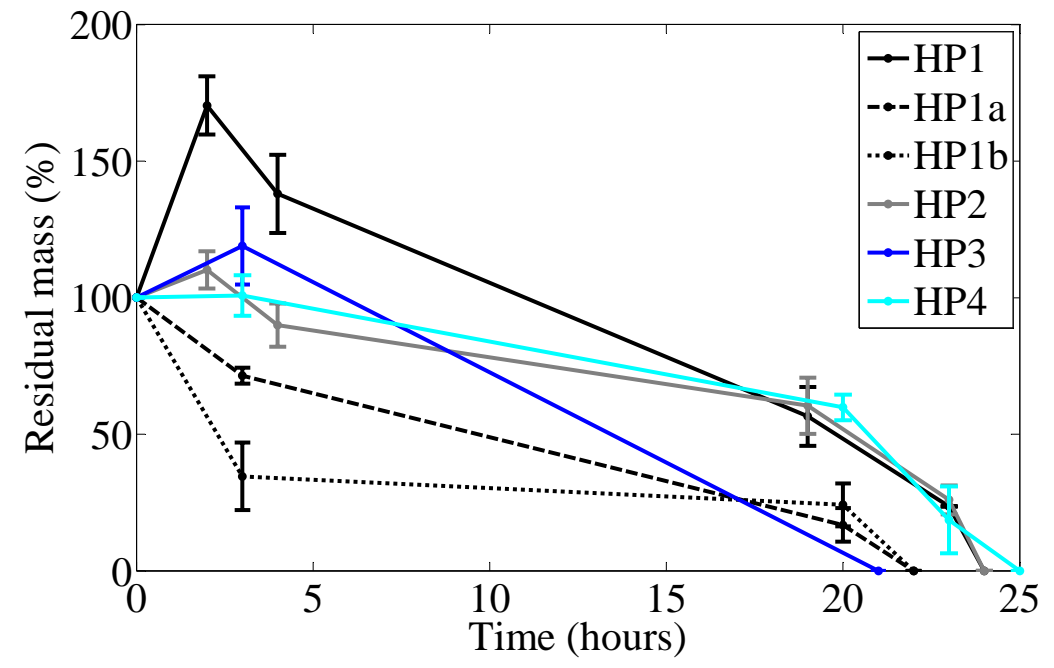

Figure 5: Enzymatic degradation profiles of the HA-PVA hydrogels in HAse enzyme (20-50 U/ml). The mean $(\mathrm{n}=3)$ and standard deviation bars are shown. 
(a)

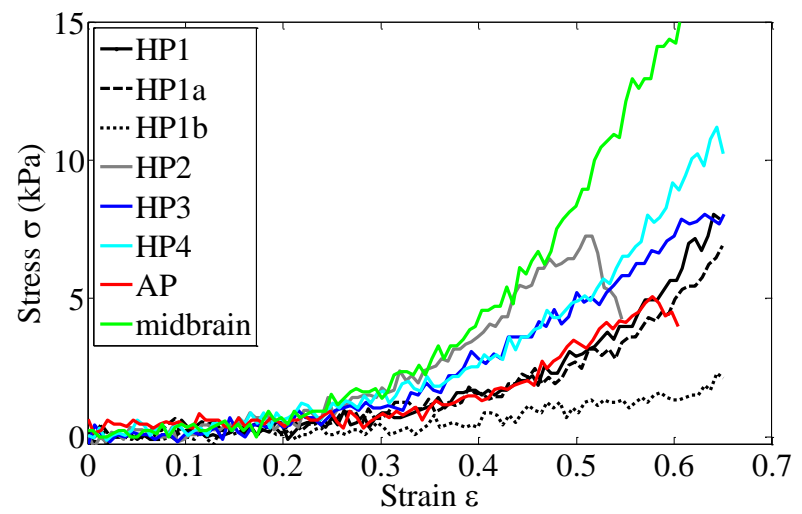

(b)

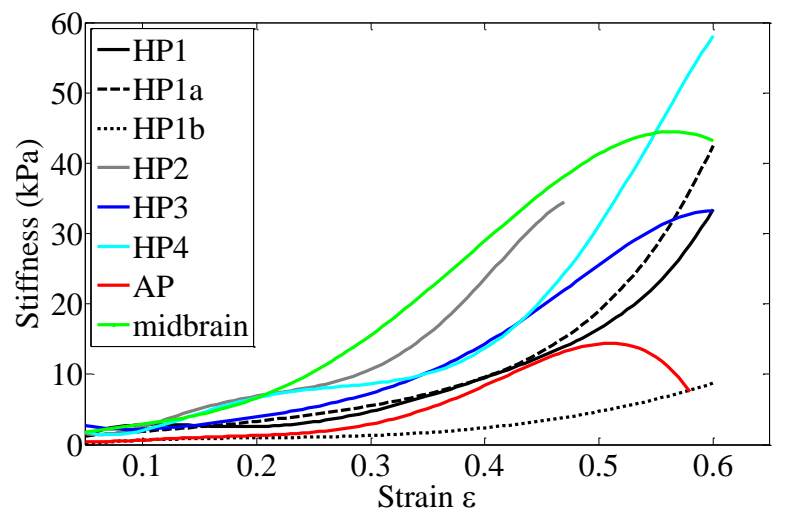

(c)

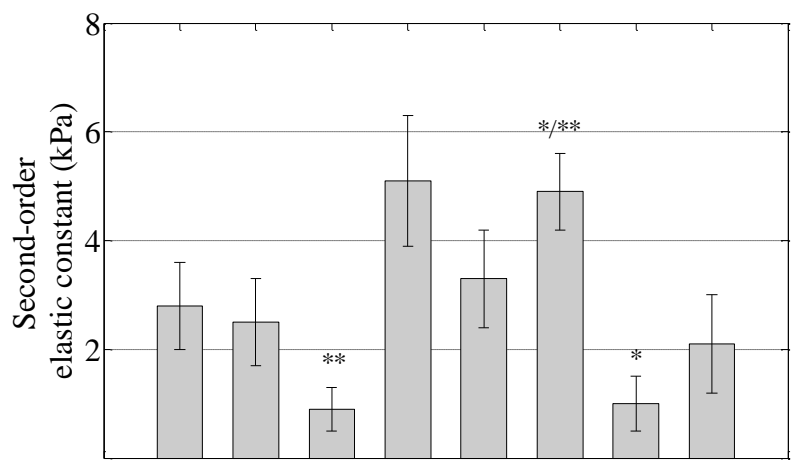

HP1 HP1aHP1b HP2 HP3 HP4 AP brain

23

Figure 6: (a) Stress as a function of strain (representative), and (b) stiffness as a function of strain curves (average), and (c) second-order elastic constants of HA-PVA and AL-PVA hydrogels and rabbit midbrain tissue. The mean $(n=3$ to 5$)$ and standard deviation bars are shown. $* \mathrm{p}<0.05$ HP4 with respect to HP1a, HP3, AP and brain, and AP with respect to HP1, HP2, HP3 and HP4. ** p < 0.01 HP1b with respect to HP1, HP1a, HP2, HP3 and HP4, and HP4 with respect to HP1 and HP1b. 

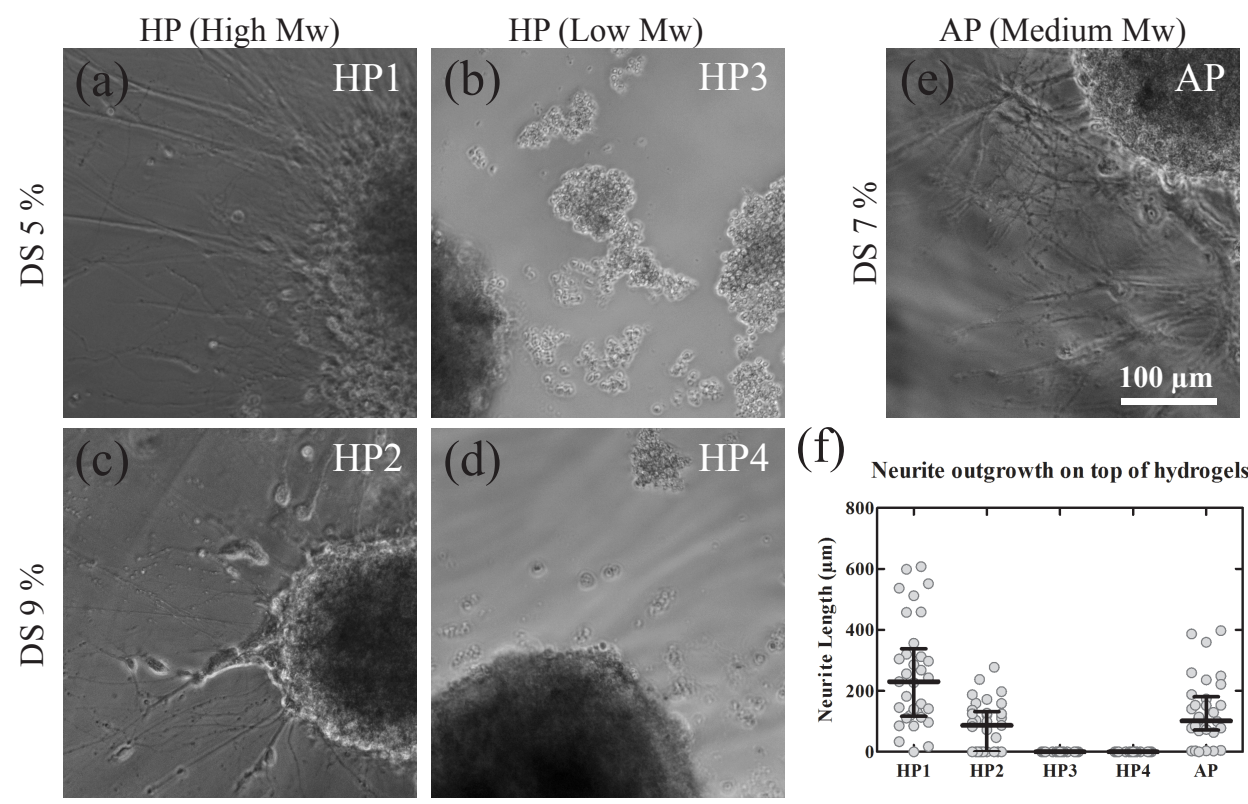

(f)

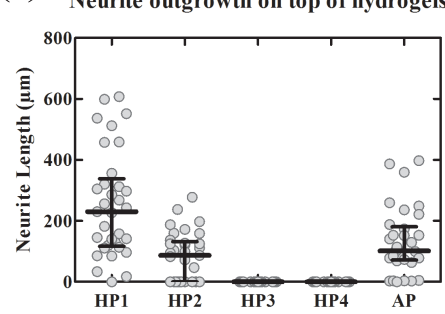

Figure 7: Representative images of neural cells growing on top of hydrogel surfaces: the high molecular weight HA hydrogels (a) HP1 and (b) HP2, the low molecular weight HA hydrogels (c) HP3 and (d) HP4 and (e) the alginate hydrogel AP. (f) Neurite outgrowth measured from cultures on top of the hydrogels. Median with inter-quartile range of the measured neurites is shown as line and whiskers whereas individual values are presented as grey dots. The scale bar in all images is $100 \mu \mathrm{m}$. 

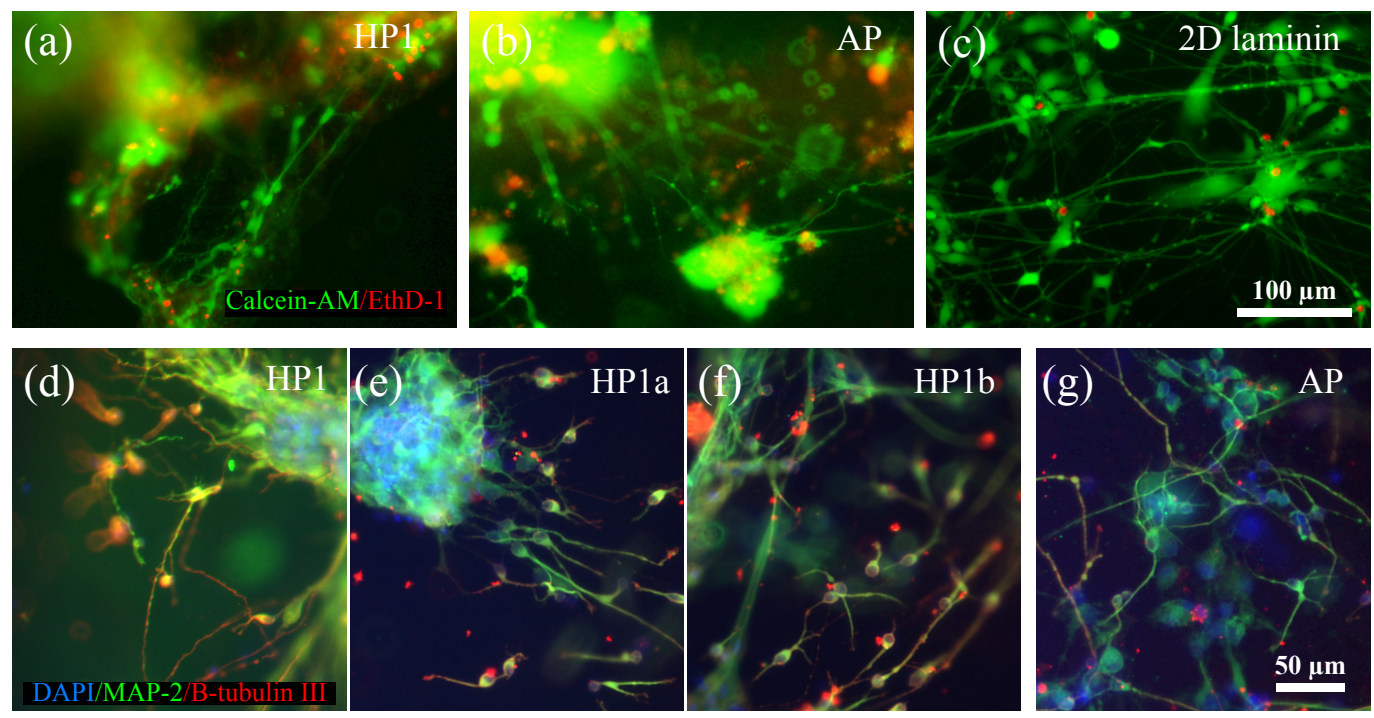

Modulus $2.8 \pm 0.8 \mathrm{kPa}$

Modulus $2.5 \pm 0.8 \mathrm{kPa}$

Modulus $0.9 \pm 0.4 \mathrm{kPa}$

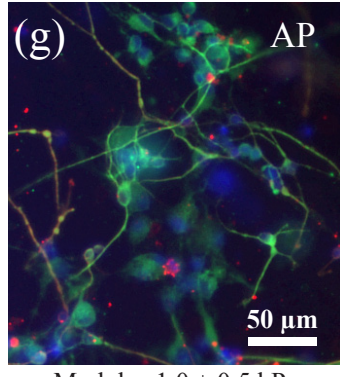

Modulus $1.0 \pm 0.5 \mathrm{kPa}$

Figure 8: Live/dead labeling of the neurons cultured two weeks inside HP1 (a) and AP (b) hydrogels, and on top of laminin coated plastic (c). Living cells are labeled with Calcein-AM (green) and dead cells with EthD-1 (red). Neurons grown for two weeks encapsulated inside the HP1 (d), HP1a (e), HP1b (f) and AP (g) hydrogels labeled with neuron specific immunocytochemical markers MAP-2 (green) and B-tubulin III (red), co-labeled with nuclear marker DAPI (blue). The second-order elastic constants of each hydrogel are shown under the images. The scale bars in images (a-c) is $100 \mu \mathrm{m}$ and in $(\mathrm{d}-\mathrm{g})$ is $50 \mu \mathrm{m}$. 